DOI: $10.17805 /$ zpu.2015.4.18

\title{
Обзор международных образовательных проектов в области послешкольного образования
}

\author{
Е. В. САБЕЛЬНИКОВА \\ (НАЦИОНАЛЬНЫЙ ИССЛЕДОВАТЕЛЬСКИЙ УНИВЕРСИТЕТ «ВЫСШАЯ ШКОЛА ЭКОНОМИКИ»), \\ Н. Л. ХМЕЛЕВА \\ (ИНСТИТУТ ГОСУДАРСТВЕННОГО АДМИНИСТРИРОВАНИЯ, Г. МОСКВА)
}

В данной статье авторы рассматривают международные образовательные исследования. Целью статьи является обзор наиболее масштабных исследовательских международных образовательных проектов в области послешкольного образования. Рассматриваются такие проекты, как «Оценка результатов обучения в высшем образовании» (AHELO), «Программа международной оценки компетенций взрослых» (PIAAC), а также «Международное исследование преподавания и обучения» (TALIS) и «Международное исследование навыков по отношению к занятости и производительности труда» (STEP).

Целью перечисленных исследований является оценка компетенций, т. е. способности применять знания и навыки в нестандартных или новых ситуациях профессионального или повседневного характера, а также факторов, влияющих на уровень компетенций. В статье описываются методология проектов, структура измерительных материалов, объем и состав выборки, реализовывался ли проект в Российской Федерации.

Результаты обзора позволяют сделать вывод о необходимости участия Российской Федерации в масштабных исследовательских международных проектах с целью включения в международные базы данных, получения возможности реализации сравнительных исследований и последующего анализа, которые позволят понять сильные и слабые стороны российской образовательной системы, оценить риски и возможности, а также использовать наилучшие международные практики и опыт при модернизации образовательного сектора. Основываясь на результатах вышеперечисленных проектов, Россия сможет более эффективно формировать и использовать необходимые компетенции, поддерживая и внедряя идею обучения взрослых людей в течение жизни, преодолевая существующие несоответствия спроса-предложения на специалистов различной квалификации.

Ключевые слова: образовательная политика; образование; компетенции; международные исследовательские образовательные проекты; ОЭCP; IEA; AHELO; PIAAC; TALIS; STEP

\section{ВВЕАЕНИЕ}

$\mathrm{H}$ аиболее крупными организациями, ведущими деятельность в области оценки образования, являются Организация экономического сотрудничества и развития (ОЭСР), Международная ассоциация по оценке учебных достижений (IEA) и Всемирный банк (The World Bank). Эти организации реализуют ряд крупномасштабных международных исследовательских образовательных проектов, которые будут рассмотрены в данной статье. В реализации каждого из проектов участвует различное количество стран, осуществляющих исследование на своей территории, но их координатором является одна из вышеперечисленных организаций, которая регулирует процессы имплементации, сбора данных и последующего анализа.

Все представленные проекты относятся к образовательной сфере и охватывают периоды начиная со школьного и заканчивая широким спектром послешкольного образования. На настоящий момент ОЭСР также делаются попытки реализации нескольких проектов в области дошкольного образования. Однако пока они находятся в стадии разработки. Исходя из вышесказанного, все имеющиеся исследования, по мнению авторов статьи, реализуемые перечисленными организациями, можно условно разделить на две категории по критерию «ступень образования». 
Первая ступень охватывает возрастной период дошкольного и школьного образования, включает такие проекты, как «Изучение качества чтения и понимания текста» (Progress in International Reading Literacy Study - PIRLS) и «Международное сравнительное мониторинговое исследование качества математического и естественнонаучного образования» (Trends in International Mathematics and Science Study - TIMMS), «Программа международной оценки образовательных достижений учащихся» (Programme for International Student Assessment - PISA). Вторая ступень включает весь период после завершения школьного образования, т. е. образование в течение последующей жизни, в ее рамках проводятся такие исследования, как «Оценка результатов обучения в высшем образовании» (Assessment of Higher Education Learning Outcomes AHELO), «Программа международной оценки компетенций взрослых» (Programme for the International Assessment of Adult Competencies - PIAAC), «Международное исследование преподавания и обучения» (Teaching and Learning International Survey TALIS), Международное исследование навыков по отношению к занятости и производительности труда (Skills Toward Employment and Productivity - STEP).

В данной статье мы рассмотрим проекты второй ступени, так как в настоящий момент образовательная политика Российской Федерации сконцентрирована на реализации стратегии обучения в течение жизни, выработка которой, в том числе, учитывает и анализ результатов вышеперечисленных проектов, на основе которых разрабатываются необходимые меры для усиления экономического роста страны в текущей сложной политической и экономической ситуации. Поэтому данный обзор представляет интерес для политиков, исследователей в области образования, так как в нем имеется основная информация по проектам, описаны методология их реализации, их значимость и возможные пути использования.

Организация экономического сотрудничества и развития (ОЭСР) является инициатором и организатором таких проектов, как PISA, AHELO, PIAAC. Исследования PIRLS и TIMMS реализуются организацией IEA (Международная ассоциация по оценке учебных достижений / International Association for the Evaluation of Educational Achievements). Часть проекта PIAAC (Образование и навыки онлайн / Education and Skills Online) реализуется ОЭСР совместно с IЕА. Базы данных, полученных в результате реализации проектов, находятся в общем доступе на сайте соответствующих организаций. Исследование STEP было реализовано Всемирным банком.

\section{ОЦЕНКА РЕЗУ АБТАТОВ ОБУЧЕНИЯ В ВЫСШЕМ ОБРАЗОВАНИИ}

\section{(ASSESSMENT OF HIGHER EDUCATION LEARNING OUTCOMES, AHELO[ES1])}

Международный проект «Оценка результатов обучения в высшем образовании» (Assessment of Higher Education Learning Outcomes, AHELO) стартовал в 2008 г. в рамках работы ОЭСР в сфере образования. Проект AHELO ставил перед собой задачу научно обоснованного измерения результатов обучения студентов. Конечной целью проекта являлась разработка комплексного подхода к оценке результатов обучения в высшем образовании, сопоставимого на международном уровне, поэтому реализованный цикл являлся пилотным (feasibility study) и предполагал оценку возможности создания такого инструмента, который позволил бы объективно оценить результаты студентов в высшем образовании в разных контекстах, включая языковой, культурный и институциональный (Мешкова, Сабельникова, 2013).

Проект AHELO изучает компетенции студентов-выпускников. Компетенции, как способность применить знания в определенной ситуации, включают необходимые на- 
выки - общие и предметно-специфические. Предметно-специфические навыки в данном проекте включают два направления: «Экономика» и «Инженерные науки». Таким образом, проект построен на исследовании трех направлений: исследование общих навыков и компетенций, исследование специальных навыков и компетенций по направлению «Экономика» и исследование специальных навыков и компетенций по направлению «Инженерные науки».

Возможность принять участие в оценке результатов обучения предоставлялась всем заинтересованным вузам страны-участницы на добровольной основе. В исследовании участвовали 17 стран, включая Россию (Countries participating ... : Электронный ресурс). Всего в тестировании приняли участие около 250 вузов и около 23000 студентов выпускных курсов вузов разных стран.

Российская Федерация в 2008-2013 гг. приняла участие в двух направлениях: «Экономика» и «Инженерные науки», в которые включились 19 и 10 вузов соответственно (Мешкова, Сабельникова, 2013). В выборку вошли следующие вузы, представленные в таблице.

Тесты для студентов представляют собой задачи, для решения которых необходимо использовать интегрированный набор навыков, включая критическое мышление, аналитическое рассуждение, решение задач и письменную коммуникацию, чтобы ответить на несколько открытых вопросов о гипотетической, но реалистичной ситуации. В ходе решения задач студентов просят не просто продемонстрировать знания, а использовать полученные в ходе обучения в вузе знания применительно к новой ситуации. Подобное построение заданий требует, чтобы молодые люди организовали доказательства из различных источников (письма, записки, резюме отчетов о научно-исследовательской работе, карты, диаграммы, таблицы и пр.). Кроме того, были использованы и задачи с множественным выбором. В целом в блок заданий были включены 6-8 заданий с конструируемым ответом и 20-25 заданий с множественным выбором.

Параллельно собирались и контекстные данные. Аля этого были разработаны три вида анкет: для студентов, преподавателей и администраторов. Также в анкету были включены вопросы, направленные на оценку самого тестирования. На этапе полевого исследования в пилотном цикле (2011 г.) тестирование и сбор информации проводились в бумажном виде, тогда как основное исследование - в электронном виде на компьютерах с доступом в Интернет.

Результаты проекта позволяют не только исследовать навыки студентов, но и показывают разницу в программах университетов, а также недостатки существующего обучения. Например, в России одной из таких сложностей стало несовершенство терминологического аппарата в экономических науках. Проект AHELO был призван обеспечить «обратную связь» о качестве результатов обучения для всех заинтересованных лиц: студентов, преподавателей, представителей учебных заведений, политиков в области образования.

Однако после завершения пилотного исследования в 2013 г. ОЭСР приостановила продолжение исследований в рамках данного проекта, поскольку в процессе проведения первого этапа было обнаружено несколько факторов, угрожающих достижению основной цели. Одним из факторов явилась значимая разница в программах обучения не только между странами, но и между вузами одной страны в пределах одного направления подготовки. Еще одной проблемой, которая характерна для такого рода исследований, стала разработка инструментария: необходимо было создать такие за- 
РОССИЙСКИЕ ВУЗЫ - УЧАСТНИКИ АНЕLО

Таблища

Table

RUSSIAN UNIVERSITIES PARTICIPATING IN AHELO

\begin{tabular}{|c|c|}
\hline Экономика & Инженерные науки \\
\hline \multicolumn{2}{|c|}{$\begin{array}{l}\text { - Северный (Арктический) федеральный университет; } \\
\text { - Северо-Восточный федеральный университет им. М. К. Аммосова; } \\
\text { - Уральский федеральный университет им. первого Президента России Б. Н. Ельцина }\end{array}$} \\
\hline $\begin{array}{l}\text { - Российская академия народного хозяйства } \\
\text { и государственной службы при Президенте } \\
\text { Российской Федерации; } \\
\text { - Государственный университет управления; } \\
\text { - Аальневосточный федеральный университет; } \\
\text { - Казанский (Приволжский) государственный } \\
\text { университет; } \\
\text { - Московский государственный институт } \\
\text { международных отношений (Университет) МИА } \\
\text { России; } \\
\text { - Московский государственный университет } \\
\text { им. М. В. Аомоносова (только этап апробации); } \\
\text { - Московский государственный университет } \\
\text { экономики, статистики и информатики; } \\
\text { - Национальный исследовательский универси- } \\
\text { тет «Высшая школа экономики»; } \\
\text { - Национальный исследовательский университет } \\
\text { «Новосибирский государственный университет»; } \\
\text { - Российский экономический университет } \\
\text { им. Г. В. Плеханова; } \\
\text { - Санкт-Петербургский государственный } \\
\text { университет; } \\
\text { - Санкт-Петербургский государственный } \\
\text { университет экономики и финансов; } \\
\text { - Сибирский федеральный университет }\end{array}$ & $\begin{array}{l}\text {-Аонской государственный технический } \\
\text { университет; } \\
\text { - Национальный исследовательский } \\
\text { Иркутский государственный технический } \\
\text { университет; } \\
\text { - Национальный исследовательский } \\
\text { Томский политехнический университет; } \\
\text { - Тюменский государственный нефтегазовый } \\
\text { университет; } \\
\text { - Уральский государственный горный } \\
\text { университет; } \\
\text { - Уральский государственный университет } \\
\text { путей сообщения; } \\
\text { - Алтайский государственный технический } \\
\text { университет им. И. И. Ползунова } \\
\end{array}$ \\
\hline
\end{tabular}

Аания, при решении которых актуализировались бы компетенции и навыки, оценка которых является основной целью, - что представляется крайне сложным, учитывая языковую, культурную и институциональную дифференциацию. Тем не менее прецедент AHELO показал принципиальную возможность оценки результатов обучения в высшем образовании, поставил новые дискуссионные вопросы, наметил дальнейшие цели и перспективы в оценке качества образования взрослых.

ПРОЕКТ «ПРОГРАММА МЕЖАУНАРОАНОЙ ОЦЕНКИ КОМПЕТЕНЦИЙ ВЗРОСАЫХ » (PROGRAMME FOR THE INTERNATIONAL ASSESSMENT OF ADULT COMPETENCIES PIAAC)

Проект «Программа международной оценки компетенций взрослых» (Programme for the International Assessment of Adult Competencies - PIAAC) был инициирован 
ОЭСР в 2008 г. с целью получения информации о ключевых компетенциях взрослого населения. Исследование ориентировано на оценку таких ключевых компетенций, как «грамотность в области чтения» (literacy), «математическая грамотность» (numeracy), «решение задач в технологически насыщенной среде» (problem solving in technology rich environment) (Шляйхер, 2010).

Выборка в каждой стране, принимавшей участие в исследовании, формировалась в соответствии с ключевыми характеристиками генеральной совокупности населения от 16 до 65 лет, проживающих на момент исследования в стране независимо от их национальности, гражданства и языкового статуса. Каждый участник решал тестовые задания, с помощью которых оценивались грамотность в области чтения, математическая грамотность и способность к решению задач в технологически насыщенной среде. Тестовые задания предъявлялись в электронном виде или в виде бумажного буклета. Кроме того, участник исследования отвечал на вопросы биографической анкеты, позволяющей собрать информацию по широкому кругу вопросов: базовые демографические характеристики и биографические данные респондента; образовательная траектория и образовательный опыт, трудовая траектория; статус на рынке труда и занятость; социальные установки и пр. (Literacy, numeracy ..., 2012; OECD skills outlook ..., 2013; Technical report ... , 2013: Электронный ресурс).

В проекте приняло участие беспрецедентное количество аналитиков, интервьюеров и респондентов из разных стран мира. В первом цикле РІААС, проведенном в 2008-2013 гг., приняли участие 24 страны, в том числе Российская Федерация. Охвачено было около 157000 взрослых респондентов из 24 стран. Второй цикл стартовал в 2012 г. и продлится до 2016 г. К нему присоединились 9 новых стран-участниц. Запланирован и третий цикл, который проводится с 2014 по 2018 г. (Literacy, numeracy ..., 2012; The survey of adult skills, 2013).

Реализация данного проекта является масштабным исследованием жизненных траекторий взрослого населения, граждан разных категорий. Результаты позволяют сделать выводы о системе образования, определить группы риска и возможности решения проблем взрослого населения, входящего в данные группы, базовые умения и навыки, необходимые человеку в определенном возрасте. На основе анализа результатов возможно сравнение уровней применения ключевых навыков, обработки информации между странами, а также сравнение уровней сформированности компетенций среди групп с различными социально-демографическими характеристиками внутри одной страны. Исследование предполагает подробное изучение факторов, связанных с развитием, формированием, поддержанием и угасанием компетенций в течение жизни, изучение связи между грамотностью взрослых, экономическими достижениями и другими социальными результатами в современном обществе и экономике.

Полученные выводы позволят выработать механизмы повышения уровня грамотности населения. Кроме того, могут быть разработаны рекомендации по повышению качества образования и развитию грамотности, на основе которых возможна организация помощи лицам с минимально необходимыми навыками, например организация курсов по овладению базовыми навыками в конкретных областях, разработка курсов повышения функциональной грамотности для безработных и т. А. Проект позволяет определить запросы населения и перспективные задачи российского общества и экономики в рамках повышения качества обучения на разных уровнях. Результаты проекта выявляют степень соответствия образовательных результатов запросам, как ин- 
дивидуальным, так и общественным, что необходимо для создания эффективной образовательной среды, и могут быть интересны как политикам, населению, так и работодателям (Образование в цифрах ... , 2014; Adults, computers ... , 2015).

\section{ПРОЕКТ «МЕЖАУНАРОАНОЕ ИССАЕАОВАНИЕ ПРЕПОААВАНИЯ И ОБУЧЕНИЯ» (TEACHING AND LEARNING INTERNATIONAL SURVEY - TALIS)}

Проект «Международное исследование преподавания и обучения» (Teaching and Learning International Survey - TALIS), реализуемый ОЭСР с 2008 г., исследует условия преподавания в школах, квалификацию и уровень профессионального развития учителей. В центре изучения - исследовательский вопрос о факторах, обусловливающих высокие результаты (высокий уровень сформированности компетенций) учащихся. B TALIS собирают и анализируют информацию об окружающей образовательной среде и методах преподавания в школах. Изучают такие условия работы учителей в школах, как профессиональное окружение, условия преподавания, подготовка учителей и их профессиональное развитие; профессиональные установки; влияние финансового вознаграждения; педагогический опыт; школьный климат; школьное лидерство (Creating effective teaching ... , 2009: Электронный ресурс; Teaching and learning ..., 2013: Электронный ресурс; TALIS 2013 technical ..., 2013: Электронный ресурс).

Российская Федерация принимает участие в реализации проекта в цикле 2013-2015 гг. Выборка исследования включила 4076 учителей средней ступени образования, 198 руководителей образовательных учреждений из 200 школ в 14 регионах России. В настоящий момент проводится анализ полученных в результате реализации исследования данных.

Результаты исследования TALIS могут быть рассмотрены как оборотная сторона образовательной системы, исследуемой в проекте PISA. Выводы предоставляют участникам и организаторам образовательного процесса возможность посмотреть на образовательные достижения учащихся с разных сторон, а именно с точки зрения влияния на достижения учащихся таких факторов, как подготовка учителей, их рабочие условия, организация образовательной среды в целом. Проект TALIS дает возможность совершенствовать стратегии развития высококачественного профессионального педагогического образования (TALIS 2013 results, 2014).

Все перечисленные проекты были реализованы или реализуются Национальным исследовательским университетом «Высшая школа экономики», который принимает активное участие в имплементации крупных международных сравнительных исследований в сфере образования (AHELO. Оценка результатов ... : Электронный ресурс; Программа международной оценки ... : Электронный ресурс; Организация и проведение ... : Электронный ресурс).

Международное исследование навыков по отношению к занятости и производительности труда (Skills Toward Employment and Productivity - STEP), проведенное Всемирным банком в 2012-2014 гг., исследует специфически значимые навыки и их связь с различными показателями рынка труда, связь между навыками и образовательными достижениями. Результаты проекта необходимы для лучшего понимания образовательной ситуации внутри стран, для элиминации неравенства и бедности, для повышения социальной включенности граждан и экономического роста (The STEP skills measurement program ... : Электронный ресурс; STEP skills measurement study, 2012: Электронный ресурс). 
Российская Федерация не принимает участия в данном проекте, так как его основной целью является оценка навыков в странах с низким и средним уровнем доходов, таких как Гана, Шри- Аанка, Вьетнам и другие. Это первая инициатива такого рода, которая позволит получить политически значимые данные для лучшего понимания запросов рынка труда на навыки, связей между приобретением навыков, образовательными достижениями и уровнем жизни, индивидуальных и социальных траекторий, сокращения неравенства и бедности, социальной интеграции и экономического роста (Youth employment programs ... , 2013; Developing skills ... , 2013; Indicators of skills ..., 2013: Электронный ресурс).

\section{ВЫВОАЫ}

Крупномасштабные международные исследовательские образовательные проекты, инициатором и организатором которых являются в основном крупные международные организации, ориентированы на различные возрастные группы населения. При этом в исследованиях и в целом в политике реализации проектов особое внимание уделяется инструментальной грамотности граждан, обеспечивающей их конкурентоспособность. Внимание к формированию навыков является важным фактором как на международном, так и на национальном уровнях. Несмотря на то что позиции России в международном масштабе довольно высоки, например, по проекту PIAAC средний балл по грамотности в области чтения составляет 275 баллов, тогда как средний по станам ОЭСР - 273 баллов, а средний балл по математической грамотности - 270 и 269 баллов соответственно (Образование в цифрах ..., , 2014; Adults, computers ..., 2015), для развития данной области необходимо иметь представление и конкретную стратегию развития навыков и формирования компетенций, а также необходимо ясно понимать, какие компетенции будут востребованы в ближайшем будущем и при долгосрочном планировании, в каком состоянии находятся требующиеся компетенции. С этой целью разработаны и реализуются несколько крупномасштабных международных проектов, призванных в соответствии с поставленными целями оценить уровень сформированности компетенций у различных групп населения.

Рассмотренные в статье проекты способствуют пониманию сильных и слабых стороны российской образовательной системы, оценке рисков и возможностей, а также имплементации наилучших международных практик и опыта при модернизации образовательного сектора. В результате продвижения политики развития навыков странами с развитой экономикой посредством реализации крупномасштабных проектов (OECD skills outlook ..., 2013; The survey of adult skills, 2013) страны с развивающейся и переходной экономикой могут использовать наилучшие мировые практики для усиления собственного экономического роста. Одной из таких глобальных мировых идей на данный момент является реализация обучения в течение жизни, что закреплено в Федеральном законе от 29 декабря 2012 г. № 273-Ф3 «Об образовании в Российской Федерации». Аля реализации такой политики, как уже было отмечено выше, необходимо понимать, какие именно компетенции востребованы или будут востребованы, а также какие несоответствия мы можем наблюдать между требованиями рынка труда и имеющимися компетенциями. Все это может обеспечить анализ данных, полученных при реализации таких исследований. 


\section{СПИСОК АИТЕРАТУРЫ}

Мешкова, Т. А., Сабельникова, Е. В. (2013) Результаты и перспективы международной оценки российского высшего образования (по итогам реализации проекта AHELO в России). М. : Федеральный институт развития образования. Вып. 12: Экономика высшей школы. 71 с.

Образование в цифрах: 2014 (2014) : краткий стат. сб. М. : Национальный исследовательский университет «Высшая школа экономики». 80 с.

Организация и проведение обследования TALIS 2013 на территории Российской Федерации [Электронный ресурс] // Институт образования НИУ ВШЭ. URL: http://ioe.hse.ru/project2014_12 [архивировано в WebCite] (дата обращения: 30.06.2015).

Программа международной оценки компетенции взрослых. Национальный-исследовательский университет «Высшая школа экономики» [Электронный ресурс] // HИУ BШЭ. URL: http://hse.ru/piaac [архивировано в WebCite] (дата обращения: 30.06.2015).

Шляйхер, А. (2010) PIAAC: новая стратегия оценки компетенций взрослых // Вопросы образования. № 1. С. 90-115.

AHELO. Оценка результатов обучения в высшем образовании [Электронный ресурс] // НИУ ВШЭ. URL: http://hse.ru/ahelo/ [архивировано в WebCite] (дата обращения: 30.06.2015).

Adults, computers and problem solving: What's the problem? (2015) / OECD Skills Studies. Paris : OECD Publishing. 188 p. DOI: 10.1787/9789264236844-en

Countries participating in the AHELO feasibility study [Электронный pecypc] // OECD.org. URL: http://oecd.org/edu/skills-beyond-school/countriesparticipatingintheahelofeasibilitystudy.htm [архивировано в WebCite] (дата обращения: 30.06.2015).

Creating effective teaching and learning environments: First results from TALIS (2009) : Executive summary // OECD.org. URL: http://oecd.org/berlin/43024880.pdf [архивировано в WebСite] (дата обращения: 30.06.2015).

Developing skills for innovative growth in the Russian Federation (2013) : Report no. ACS1549 / K. Vasiliev, S. Roshchin, I. O. Maltseva, P. Travkin, A. Lukiyanova, D. Chugunov, I. Shulga, J. J. Rutkowski, P. M. Cahu, S. Nellemann. Washington, DC : The World Bank. 150 p.

Indicators of skills for employment and productivity: A conceptual framework and approach for low-income countries (2013) : A report for the Human Resource Development Pillar of the G20 Multiyear Action Plan on Development / OECD and the World Bank in collaboration with ETF, ILO and UNESCO [Электронный ресурс] // OECD.org. URL: http://oecd.org/g20/topics/development/ indicators-of-skills-employment-and-productivity.pdf [архивировано в WebCite] (дата обращения: 30.06.2015).

Literacy, numeracy and problem solving in technology-rich environments (2012): Framework for the OECD survey of adult skills. Paris : OECD Publishing. 80 p. DOI: 10.1787/9789264128859-en

OECD skills outlook 2013 (2013) : First results from the survey of adult skills. Paris : OECD Publishing. 464 p. DOI: 10.1787/9789264204256-en

STEP skills measurement study. (2012) [Электронный ресурс]// The World Bank. URL: http:// siteresources.worldbank.org/EXTHDOFFICE/Resources/5485726-1281723119684/STEP_Skills Measurement_Brochure_Jan_2012.pdf [архивировано в WebCite] (дата обращения: 12.01.2015).

TALIS $20 \overline{13}$ results $(\overrightarrow{2014)} \overline{)}$ : An international perspective on teaching and learning. Paris : OECD Publishing. 440 p. DOI: 10.1787/9789264196261-en

TALIS 2013 technical report (2013) : Teaching and learning international survey [Электронный pecypc] // OECD.org. URL: http://oecd.org/edu/school/TALIS-technical-report-2013.pdf [архивировано в WebCite] (дата обращения: 30.06.2015).

Teaching and learning international survey TALIS 2013 (2013) : Conceptual framework [Электронный ресурс] // OECD.org. URL: http://oecd.org/edu/school/TALIS\%20Conceptual\%20Framework_FINAL.pdf [архивировано в WebCite] (дата обращения: 30.06.2015).

Technical report of the survey of adult skills (PIAAC). (2013) [Электронный ресурс] // OECD.org URL: http://oecd.org/site/piaac/_Technical\%20Report_17OCT13.pdf [архивировано в WebCite] (дата обращения: 30.06.2015). 
The STEP skills measurement program [Электронный ресурс]// The Microdata Library. URL: http://microdata.worldbank.org/index.php/catalog/step/about\%20 [архивировано в WebCite] (дата обращения: 30.06.2015).

The survey of adult skills (2013) : Reader's companion. Paris : OECD Publishing. 124 p. DOI: 10.1787/9789264204027-en

Youth employment programs: An evaluation of World Bank and IFC support. (2013) Washington, DC : World Bank Group. xxxiii, 173 p. DOI: 10.1596/978-0-8213-9794-7

Аата поступления: 1.07.2015 2.

\section{INTERNATIONAL PROJECTS IN POST-SECONDARY EDUCATION: A REVIEW ARTICLE \\ E. V. SABELNIKOVA \\ (NATIONAL ReSEARCH UNIVERSITY "HIGHER SCHOOL OF ECONOMICS"), \\ N. L. KHMELEVA \\ (INSTITUTE of State AdMINISTRATION, Moscow)}

In this article, we propose a classification of international research educational surveys based on the criterion of the "level of education": secondary or post-secondary. Our aim was to review largescale international research surveys in the sphere of post-secondary education, such as Assessment of Higher Education Learning Outcomes (AHELO), Programme for the International Assessment of Adult Competencies (PIAAC), Teaching and Learning International Survey (TALIS), Skills toward Employment and Productivity (STEP).

An important component of these surveys is the assessment of competencies, i.e. the students' ability to apply their knowledge and skills in non-standard situations, including those new to them professionally and found in everyday life, as well as the assessment of factors which contribute to the competency level. The article describes the methodology of the projects, the materials used for testing, the structure of the sample and whether a particular project has been implemented in the Russian Federation.

The outcomes of the review allowed us to conclude that for Russia, participating in large-scale international research educational surveys is a must. Among reasons for participation are the inclusion of Russian data into international databases and an opportunity for comparative studies and further analysis. These activities will help understand the strengths and weaknesses of the education system in Russia, assess its opportunities and threats, and apply the best international practices and experience in the modernization of the education sector. By learning from the outcomes of the abovementioned surveys, Russia will be able to act more effectively in developing and using the relevant competencies, involving people of all ages in education, popularizing lifelong learning to achieve the supply and demand equilibrium in the quality and quantity of various types of professionalized labor force.

Keywords: educational policy; education; competencies; international re-search educational surveys; OECD; IEA; AHELO; PIAAC; TALIS; STEP.

\section{REFERENCES}

Meshkova, T. A. and Sabelnikova, E. V. (2013) Rezul'taty i perspektivy mezhdunarodnoi otsenki rossiiskogo vysshego obrazovaniia (po itogam realizatsii proekta AHELO $v$ Rossii) [Results and prospects of international assessment of Russian higher education (Following the results of AHELO implementation in Russia)]. Moscow, Federal Institute of Education Development Publ. Vol. 12: Ekonomika vysshei shkoly [Economics of the higher school]. 71 p. (In Russ.).

Obrazovanie v tsifrakh: 2014 [Education in figures: 2014] (2014) : concise statistical digest. Moscow, National Research University "Higher School of Economics" Publ. 80 p. (In Russ.).

Organizatsiia i provedenie obsledovaniia TALIS 2013 na territorii Rossiiskoi Federatsii [Organisation and implementation of TALIS 2013 in the Russian Federation]. Institut obrazovaniia NIU VShE [Institute of Education of the National Research University "Higher School of Economics"] 
[online] Available at: http://ioe.hse.ru/project2014_12 [archived in WebCite] (accessed 30.06.2015). (In Russ.).

Programma mezhdunarodnoi otsenki kompetentsii vzroslykh [Programme for the International Assessment of Adult Competencies]. NIU VSbE [National Research University "Higher School of Economics"] [online] Available at: http://hse.ru/piaac [archived in WebCite] (accessed 30.06.2015). (In Russ.).

Schleicher, A. (2010) PIAAC: novaia strategiia otsenki kompetentsii vzroslykh [PIAAC: A new strategy for assessing adult competencies]. Voprosy obrazovaniia, vol. 1, pp. 90-115. (In Russ.).

AHELO. Otsenka rezul'tatov obucheniia v vysshem obrazovanii. [AHELO. Assessment of higher education learning outcomes]. NIU VSbE [National Research University "Higher School of Economics"] [online] Available at: http://hse.ru/ahelo/ [archived in WebCite] (accessed 30.06.2015). (In Russ.).

Adults, computers and problem solving: What's the problem? (2015)/ OECD Skills Studies. Paris, OECD Publishing. 188 p. DOI: 10.1787/9789264236844-en

Countries participating in the AHELO feasibility study. OECD.org [online] Available at: http://oecd.org/edu/skills-beyond-school/countriesparticipatingintheahelofeasibilitystudy.htm [archived in WebCite] (accessed 30.06.2015).

Creating effective teaching and learning environments: First results from TALIS (2009) : Executive summary. OECD.org. [online] Available at: http://oecd.org/berlin/43024880.pdf [archived in Web Cite] (accessed 30.06.2015).

Developing skills for innovative growth in the Russian Federation (2013) : Report no. ACS1549 / K. Vasiliev, S. Roshchin, I. O. Maltseva, P. Travkin, A. Lukiyanova, D. Chugunov, I. Shulga, J. J. Rutkowski, P. M. Cahu, S. Nellemann. Washington, DC, The World Bank. 150 p.

Indicators of skills for employment and productivity: A conceptual framework and approach for low-income countries (2013) : A report for the Human Resource Development Pillar of the G20 Multiyear Action Plan on Development / OECD and the World Bank in collaboration with ETF, ILO and UNESCO. OECD.org [online] Available at: http://oecd.org/g20/topics/development/indicators-ofskills-employment-and-productivity.pdf [archived in WebCite] (accessed 30.06.2015).

Literacy, numeracy and problem solving in technology-rich environments (2012): Framework for the OECD survey of adult skills. Paris, OECD Publishing. 80 p. DOI: 10.1787/9789264128859-en

OECD skills outlook 2013 (2013) : First results from the survey of adult skills. Paris, OECD Publishing. 464 p. DOI: 10.1787/9789264204256-en

STEP skills measurement study. (2012) The World Bank [online] Available at: http://siteresources.worldbank.org/EXTHDOFFICE/Resources/5485726-1281723119684/STEP_Skills Measurement_Brochure_Jan_2012.pdf [archived in WebCite] (accessed 12.01.2015).

TALIS $20 \overline{1} 3$ results $\overrightarrow{(201 \overline{4})}$ : An international perspective on teaching and learning. Paris, OECD Publishing. 440 p. DOI: 10.1787/9789264196261-en

TALIS 2013 technical report (2013) : Teaching and learning international survey. OECD.org [online] Available at: http://oecd.org/edu/school/TALIS-technical-report-2013.pdf [archived in WebCite] (accessed 30.06.2015).

Teaching and learning international survey TALIS 2013 (2013) : Conceptual framework. OECD.org [online] Available at: http://oecd.org/edu/school/TALIS\%20Conceptual\%20Framework_FINAL.pdf [archived in WebCite] (accessed 30.06.2015).

Technical report of the survey of adult skills (PIAAC). (2013) OECD.org [online] Available at: http://oecd.org/site/piaac/_Technical\%20Report_17OCT13.pdf [archived in WebCite] (accessed 30.06.2015).

The STEP skills measurement program. The Microdata Library. [online] Available at: http:// microdata.worldbank.org/index.php/catalog/step/about\%20 [archived in WebCite] (accessed 30.06.2015).

The survey of adult skills (2013) : Reader's companion. Paris, OECD Publishing. 124 p. DOI: 10.1787/9789264204027-en 
Youth employment programs: An evaluation of World Bank and IFC support. (2013) Washington, DC : World Bank Group. xxxiii, 173 p. DOI: 10.1596/978-0-8213-9794-7

Submission date: 1.07.2015.

Сабельникова Елена Владимировна - заместитель директора Информационно-координационного центра по взаимодействию с Организацией экономического сотрудничества и развития Института статистических исследований и экономики знаний Национального исследовательского университета «Высшая школа экономики». Адрес: 101000, Россия, г. Москва, ул. Мясницкая, д. 20. Тел.: +7 (495) 772-95-90. Эл. адрес: esabelnikova@hse.ru

SPIN-код РИНЦ: 6144-5367; ORCID: 0000-0002-0261-1345; ResearcherID: B-7855-2014.

Хмелева Наталья $\Lambda$ ьвовна - старший преподаватель кафедры психологии и педагогики психолого-педагогического факультета Института государственного администрирования (Москва). Адрес: 119261, Россия, г. Москва, Аенинский пр-т, А. 80. Тел.: +7 (499) 131-99-79. Эл. aspec:nlh2009@yandex.ru

Sabelnikova Elena Vladimirovna, Deputy Director, OECD-HSE Partnership Centre, Institute for Statistical Studies and Economics of Knowledge, National Research University "Higher School of Economics". Postal address: 20 Myasnitskaya St., 101000 Moscow, Russian Federation. Tel.: +7 (495) 772-95-90. E-mail: esabelnikova@hse.ru

Khmeleva Natalia Lvovna, Senior Lecturer, Department of Psychology and Pedagogy, Faculty of Psychology and Pedagogy, Institute of State Administration, Moscow. Postal address: 80 Leninsky Avenue, 119261 Moscow, Russian Federation. Tel.: +7 (499) 131-91-88. E-mail:nlh2009@yandex.ru 